ISSN = 1980-993X - doi:10.4136/1980-993X
www.ambi-agua.net
E-mail: ambi-agua@agro.unitau.br
Tel.: (12) 3625-4212

\title{
Ictiofauna de igarapés de pequenas bacias de drenagem em área agrícola do Nordeste Paraense, Amazônia Oriental
}

\author{
(http://dx.doi.org/10.4136/ambi-agua.739)
}

\author{
Jean Michel Corrêa ${ }^{1}$; Pedro Gerhard ${ }^{2}$; Ricardo de Oliveira Figueiredo ${ }^{3}$ \\ ${ }^{1}$ Instituto de Geociências, Universidade Federal do Pará, Belém, \\ e-mail: jeanoceano@yahoo.com.br, \\ ${ }^{2}$ Embrapa Amazônia Oriental, Belém, e-mail: pgerhard@cpatu.embrapa.br, \\ ${ }^{3}$ Embrapa Meio Ambiente, Jaguariúna, e-mail: ricfig@cnpma.embrapa.br
}

\section{RESUMO}

Comunidades de peixes podem se distribuir no espaço e no tempo de maneira organizada, seguindo um padrão que pode ser percebido pela associação ou agrupamento das espécies e pela relação de algumas espécies com determinados habitats. O número reduzido de estudos e o pouco conhecimento da fauna aquática na Amazônia resultam em subestimativas da diversidade da ictiofauna de igarapés (riachos amazônicos). No presente estudo, em três microbacias predominantemente ocupadas por agricultura familiar, foram coletados 2.117 peixes, distribuídos em sete ordens, 13 famílias, 27 gêneros e 43 espécies. A espécie mais abundante em todas as amostras coletadas foi Hyphessobrycon heterorhabdus, com 337 indivíduos, seguido por Bryconops melanurus, com 326 indivíduos. A riqueza de espécies foi maior num trecho do Igarapé Pachibá (IGPA-B), com 21 espécies. O Índice de Dominância de Simpson mostrou o valor mais alto no trecho B do Igarapé Cumaru, com valor 0,43, enquanto o Índice de Diversidade de Shannon revelou que o IGPA-B possuiu a maior diversidade, com valor 2,39. Iguanodectes rachovii foi a espécie amostrada com mais constância, e ocorreu em 50\% das amostras. Os resultados demonstraram que trechos médios dos igarapés apresentam maior diversidade de espécies, respondendo ao tamanho do habitat. Neste estudo foi possível observar que microbacias agrícolas dominadas por agricultura de pequeno porte pode suportar uma diversidade de peixes de igarapés razoável.

Palavras-chave: peixes de riachos, diversidade, agricultura familiar.

\section{Fish fauna of streams of small watersheds in agriculture area of northeastern Pará, Eastern Amazon}

\begin{abstract}
Stream fish communities can show spatial and temporal patterns, which can be revealed by species association or species groups as well as by species preference to certain habitats. The reduced number of studies and the scarce knowledge on the aquatic fauna in the Amazon results on an under-estimative upon stream fish fauna diversity. In this study, a survey at three small Amazonian catchments located in family agriculture areas, a total of 2,117 individuals were collected, belonging to seven orders, 13 families, 27 genera and 43 species. The most abundant species in all samples was Hyphessobrycon heterorhabdus, totaling 337 individuals, followed by Bryconops melanurus, with 326 individuals. The species richness was highest in a Pachibá stream reach, with 21 species. Simpson's diversity index in a Cumaru stream reach showed the highest species dominance with a value of 0.43 , while Shannon's diversity index showed that the Pachibá stream lowest reach, had the greatest diversity with a value of 2.39.
\end{abstract}


Iguanodectes rachovii was the most constant species, occurring in 50\% of samples. Therefore, as a response to habitat size, the intermediate stream reaches presented the highest species diversity. Our study showed that agricultural catchments dominated by smallholder farmers can bear a reasonable stream fish diversity.

Keywords: stream fishes, diversity, family agriculture.

\section{INTRODUÇÃO}

A conservação da biodiversidade em ecossistemas aquáticos é um dos desafios mais importantes e difíceis a serem enfrentados no mundo atual (Chernoff et al., 1996). Esse desafio não pode ser superado sem conhecimento detalhado da diversidade taxonômica e padrões de distribuição das espécies. Comunidades tropicais de animais são caracteristicamente muito diversificadas, com grande número de espécies e interações complexas, quando comparadas com comunidades localizadas em zonas temperadas. A fauna de peixes segue esta regra ecológica geral, tanto dentro de famílias quanto de ambientes (Lowe-McConnell, 1999). A fauna de peixes de água doce localizada na região Neotropical é mais diversificada e rica do planeta, com mais de 4.475 espécies descritas (Reis et al., 2003).

Os igarapés, denominação regional dada aos riachos amazônicos, são cursos d'água de pequeno porte, caracterizados pelo leito delimitado, correnteza relativamente acentuada e baixa temperatura da água. Sua porção média e superior é quase totalmente encobertas pelo dossel da floresta ripária, e seu leito tipicamente contém acúmulo de troncos e galhos caídos. Uma característica física dos igarapés é a subida abrupta do nível da água durante a ocorrência de fortes chuvas em suas bacias de drenagem. Nesses eventos meteorológicos extremos o nível da água alcança ou ultrapassa os limites das margens, disponibilizando aos peixes novos recursos alimentares. Em decorrência da redução da luz incidente produzido pela sombra das espécies florestais e a correnteza relativamente acentuada, os igarapés são sistemas aquáticos com baixa produtividade biológica e bastante dependentes da floresta. Esta atua como fonte de recursos alimentares para o sistema lótico, os quais são a base da cadeia trófica nestes ecossistemas (Santos e Ferreira, 1999).

As atividades agrícolas e pecuárias na bacia hidrográfica de um pequeno igarapé podem determinar uma série de alterações no ambiente aquático. A movimentação do solo nas vertentes da bacia para preparo de cultivos, a adição de corretivos, fertilizantes e agroquímicos, a compactação do solo pelo gado e a retirada de vegetação nativa na zona ripária são alguns destes determinantes. Os possíveis efeitos diretos incluem: aporte elevado de sedimentos no canal, alterações no regime de vazão do igarapé, na qualidade química e física da água, na entrada de energia solar direta e na entrada de resíduos de substâncias complexas potencialmente tóxicas. Indiretamente, esses efeitos determinam impactos negativos sobre a estrutura física e química do habitat dos peixes. Estes e outros aspectos foram estudados em trabalhos recentes, como Buss et al. (2002), Allan (2004), Ferreira e Casatti (2006), Casatti (2010), De Paula et al. (2011), Machado et al. (2011).

O objetivo desse trabalho foi analisar a composição e diversidade da ictiofauna em três pequenos igarapés (Cumaru, São João e Pachibá) situados no nordeste paraense, cujas bacias são ocupadas predominantemente pela agricultura familiar.

\section{MATERIAL E MÉTODOS}

\section{1. Área de estudo}

As três microbacias estudadas localizam-se no nordeste do Estado do Pará. As microbacias dos igarapés Cumaru e São João pertencem a bacia do Rio Maracanã, enquanto a 
CORRÊA, J. M.; GERHARD, P.; FIGUEIREDO, R. O. Ictiofauna de igarapés de pequenas bacias de drenagem em área agrícola do Nordeste Paraense, Amazônia Oriental. Ambi-Agua, Taubaté, v. 7, n. 2, p. 214-230, 2012. (http://dx.doi.org/10.4136/ambi-agua.739)

microbacia do igarapé Pachibá, afluente do igarapé Timboteua, pertence a bacia do Rio Marapanim (Figura 1). A agricultura familiar é muito expressiva nessa região, cujas principais culturas são o milho, o caupi, e a mandioca, além de cultivos industriais, como o maracujá e a pimenta-do-reino (Sommer et al., 2004). Além desta cobertura vegetal agrícola, existem importantes áreas de florestas secundárias em diferentes estágios sucessionais, as quais ocupam também os setores marginais aos cursos de água, constituindo floresta ripária. No entanto, essas florestas ripárias vêm sendo desmatadas no processo de expansão das áreas de cultivo e para retirada de madeira para as pequenas propriedades rurais da região (Watrin et al., 2009).

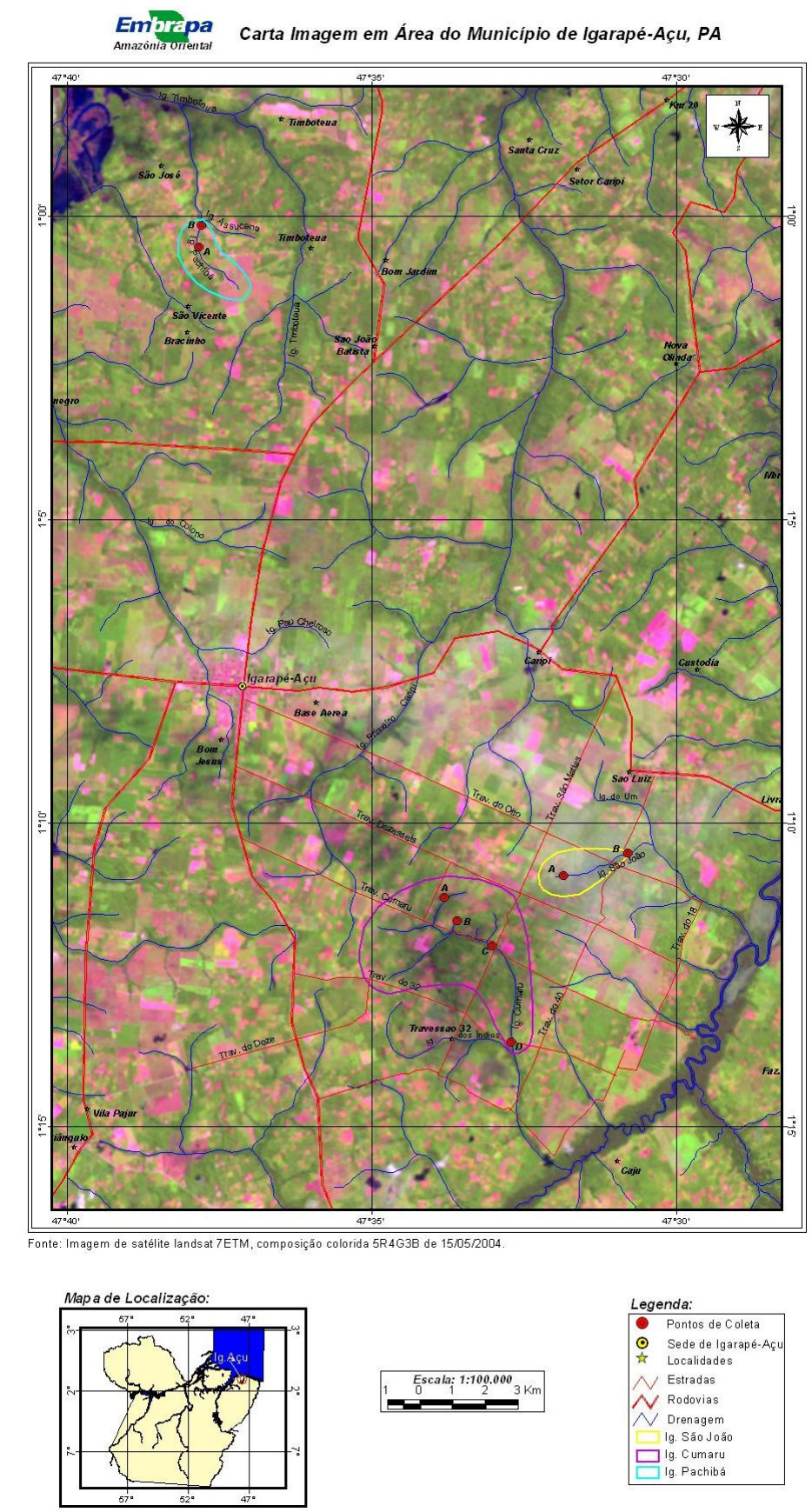

Figura 1. Localização das microbacias dos igarapés Cumaru, São João e Pachibá (A, B, C e D, representam estações de amostragem).

Fonte: Laboratório de Sensoriamento Remoto da EMBRAPA Amazônia Oriental. 
CORRÊA, J. M.; GERHARD, P.; FIGUEIREDO, R. O. Ictiofauna de igarapés de pequenas bacias de drenagem em área agrícola do Nordeste Paraense, Amazônia Oriental. Ambi-Agua, Taubaté, v. 7, n. 2, p. 214-230, 2012. (http://dx.doi.org/10.4136/ambi-agua.739)

Oito estações de amostragem foram definidas nas três microbacias estudadas pelo Projeto Agrobacias Amazônicas, desenvolvido pela Embrapa Amazônia Oriental, no qual uma série de outros estudos em temas como Biogeoquímica e Impactos Ambientais, por exemplo, foram abordados. Essas oito estações, localizadas em área de agricultura familiar, foram assim alocadas: quatro estações no igarapé Cumaru, sendo duas localizadas em áreas de nascentes e duas no trecho médio do canal principal; duas estações no igarapé São João, sendo uma em área de nascente e outra no trecho médio do canal principal; e duas estações localizadas no igarapé Pachibá, uma mais próxima à nascente e outra no trecho médio. A escolha de mais estações de amostragem na microbacia do Cumaru deve-se ao fato desta possuir uma área bem maior do que as demais microbacias avaliadas.

Gerhard (2007) realizou trabalho de classificação da paisagem das bacias aqui estudadas, utilizando imagens Landsat, dados do IBGE e vários programas computacionais em sensoriamento remoto, que proporcionaram inclusive as estimativas de áreas apresentadas na Tabela 1. Ressalta-se que os cursos d'água estudados são de primeira ordem (nascentes) a segunda e terceira ordens (trechos médios).

Tabela 1. Estações de amostragem, com localizações, microbacias, coordenadas geográficas e as áreas dos trechos amostrados.

\begin{tabular}{|c|c|c|c|c|}
\hline Estação & Microbacia & & Localização & Área (ha) \\
\hline IGCU-A & Cumaru & nascente & $01^{\circ} 11^{\prime} 25,0^{\prime \prime}$ S e $47^{\circ} 34^{\prime} 00,9^{\prime \prime} \mathrm{W}$ & 11,20 \\
\hline IGCU-B & Cumaru & nascente & $01^{\circ} 11^{\prime} 36,2^{\prime \prime}$ 'S e $47^{\circ} 33^{\prime} 39,8^{\prime \prime} \mathrm{W}$ & 8,62 \\
\hline IGCU-C & Cumaru & trecho médio & $01^{\circ} 12^{\prime} 00,8^{\prime \prime}$ S e $47^{\circ} 33^{\prime} 04,3^{\prime \prime} \mathrm{W}$ & 1180,28 \\
\hline IGCU-D & Cumaru & trecho médio & $01^{\circ} 13^{\prime} 31,0^{\prime \prime} \mathrm{S}$ e $47^{\circ} 32^{\prime} 46,3^{\prime \prime} \mathrm{W}$ & 1849,68 \\
\hline IGSJ-A & São João & nascente & $01^{\circ} 10^{\prime} 47,7^{\prime \prime}$ S e $47^{\circ} 32^{\prime} 35,5^{\prime \prime} \mathrm{W}$ & 181,73 \\
\hline IGSJ-B & São João & trecho médio & $01^{\circ} 10^{\prime} 30,0^{\prime \prime} \mathrm{S}$ e $47^{\circ} 30^{\prime} 56,1^{\prime \prime} \mathrm{W}$ & 570,51 \\
\hline IGPA-A & Pachibá & nascente & $01^{\circ} 00^{\prime} 24,2^{\prime \prime}$ S e $47^{\circ} 37^{\prime} 58,8^{\prime \prime} \mathrm{W}$ & 200,03 \\
\hline IGPA-B & Pachibá & trecho médio & $01^{\circ} 00^{\prime} 08,2^{\prime \prime}$ S e $47^{\circ} 37^{\prime} 53,3^{\prime \prime} \mathrm{W}$ & 322,60 \\
\hline
\end{tabular}

\subsection{Método de coleta}

As campanhas para estudo da ictiofauna foram realizadas em nove diferentes ocasiões com a finalidade de contemplar três condições diferentes, ajustando ao ciclo natural das chuvas e regimes hidrológicos da região Amazônica (Bastos e Pacheco, 2000; Pacheco e Bastos, 2007). Dessa maneira, foram realizadas: (A) três campanhas de campo realizadas no período de chuva, em maio e junho de 2006 e em março de 2007, (B) três amostragens no período de estiagem, em outubro e novembro de 2006 e em janeiro de 2007 e (C) três amostragens no período de transição entre os dois períodos citados, em julho, agosto e setembro de 2006. Os peixes foram coletados apenas no período diurno.

Em cada estação foi utilizado como apetrecho de pesca uma tarrafa com malha de $5 \mathrm{~mm}$, diâmetro de $3 \mathrm{~m}$ e uma rede de arrasto com comprimento de $6 \mathrm{~m}$, altura de $1,2 \mathrm{~m}$ e malha de $5 \mathrm{~mm}$. A captura foi feita por duas pessoas. Também foi utilizado puçá com malha de $2 \mathrm{~mm}$ para capturar peixes de menor porte. Para evitar a fuga dos cardumes durante a coleta, o trecho de coleta foi bloqueado por redes de espera com malha de $5 \mathrm{~mm}$ entre nós opostos, comprimento de $2 \mathrm{~m}$ e altura de $1,2 \mathrm{~m}$ em suas extremidades. Foram realizadas perturbações na água a fim de desalojar espécimes que ficam escondidas junto as margens. Foram utilizados covos feitos de garrafas de "pet" de dois litros de volume, que eram presos em pequenas estacas na margem do igarapé. Dentro dos covos foram colocadas pequenas 
quantidades de ração para facilitar a atração e captura dos peixes. A duração de cada coleta em cada ponto foi de uma hora, somando-se os apetrechos de pesca. Os indivíduos capturados foram colocados em sacos plásticos, etiquetados e acondicionados em caixas de isopor com gelo. No laboratório, o material resfriado foi colocado em frascos etiquetados para identificação, e conservados "in natura" em um freezer.

No momento da identificação das espécies, foram empregadas chaves de identificação dicotômicas pertinentes aos grandes grupos, como por exemplo, chaves de identificação para gênero e espécie contida em Ferreira et al. (1998), catálogos de espécies de importância comercial como o de Silvano et al. (2001), Santos et al. (2004) e na base de dados Fishbase (Froese e Pauly, 2007).

\subsection{Análise de dados}

Para comparar as assembléias de peixes em termos de atributos ecológicos (Krebs, 1999), utilizou-se o índice de constância (C) de espécies, usando a seguinte equação:

$$
C=\frac{c_{i}}{c}
$$

em que :

$c_{i}$ é o número de coletas contendo a espécie $i$ e $c$ é o número total de coletas efetuadas.

Uma espécie é considerada constante quando $\mathrm{C}>50 \%$ das capturas. Se $50 \% \geq \mathrm{C} \geq 25 \%$, é considerada acessória e acidental quando $\mathrm{C}<25 \%$.

A fim de verificar a dominância de espécies nas estações de amostragem, o índice de dominância $(D)$ para cada estação de amostragem foi calculado, utilizando-se a equação de Simpson:

$$
D=\sum\left(p_{i}\right)^{2}
$$

em que :

$p_{i}$ é a proporção de indivíduos da espécie $i$ em relação ao número total de indivíduos da amostra. Este índice atribui um peso maior às espécies abundantes.

O índice de diversidade de Shannon $\left(H^{\prime}\right)$ está relacionado com a riqueza de espécies, atribuindo um peso maior às espécies raras. Esse índice foi calculado para cada estação de amostragem usando a equação de Shannon:

$$
H^{\prime}=-\Sigma\left(p_{i}\right) x \ln \left(p_{i}\right)
$$

A eqüitabilidade de Shannon $(E q)$ revela uma uniformidade na distribuição das espécies, ou seja, se os indivíduos das espécies da amostra distribuem-se em proporções mais ou menos similares. Este índice foi calculado conforme a equação:

$$
E q=\frac{H^{\prime}}{H_{\max }^{\prime}}
$$

em que:

H'é o índice de diversidade de Shannon e H'max é a diversidade máxima de Shannon, calculada por:

$$
H^{\prime} \max =\ln S
$$

em que:

$S$ é o número de espécies da amostra. 
A fim de compreender a similaridade entre as comunidades de peixes amostradas nas oito estações, foi calculada a similaridade percentual (ou medida de Renkonen) (Krebs, 1999). Os valores resultantes entre os pares de amostras das estações foram dispostos em uma matriz de dissimilaridade, calculada como a recíproca da similaridade. Esta matriz de dissimilaridades foi submetida a uma análise de agrupamento, utilizando como método de ligação o algoritmo UPGMA (Unweighted Pair Group Method of Arithmetic Means).

$\mathrm{O}$ número de espécies que são encontradas durante um programa de amostragem depende, entre outros fatores, do tamanho da amostra. A fim de verificar se o esforço amostral empregado está próximo a um número máximo de espécies, uma curva de acumulação de espécies ou do coletor foi plotada para cada estação de amostragem, considerando os nove meses de coletas.

A relação entre as variáveis ecológicas e as variáveis relacionadas ao uso da terra foram submetidos a uma análise de regressão linear simples. É importante ressaltar que nem todas as variáveis ecológicas foram utilizadas e que foi preciso fazer uma matriz de correlação de Pearson para selecionar as variáveis a serem utilizadas. As análises estatísticas foram feitas utilizando-se o aplicativo STATISTICA 5.0 ${ }^{\mathrm{TM}}$.

\title{
3. RESULTADOS E DISCUSSÃO
}

\subsection{Composição ictiofaunística}

O número total de indivíduos coletados neste trabalho foi de 2.117. Ao todo foram identificados 43 táxons, pertencentes a 27 gêneros, 13 famílias e sete ordens. A lista taxonômica para as espécies do local de estudo foi elaborada com base na classificação sistemática proposta em Buckup e Menezes (2003), como segue:

\author{
Classe ACTINOPTERYGII \\ Ordem CHARACIFORMES \\ Família ANOSTOMIDAE \\ Leporinus sp. \\ Família CHARACIDAE \\ Astyanax bimaculatus (Linnaeus, 1758) \\ Astyanax sp. 1 \\ Astyanax sp. 2 \\ Astyanax sp. 3 \\ Astyanax sp. 4 \\ Bryconops melanurus (Bloch, 1795) \\ Bryconops sp. 1 \\ Bryconops sp. 2 \\ Hemigrammus sp. 1 \\ Hemigrammus sp. 2 \\ Hyphessobrycon cf. bentosi Durbin, 1908 \\ Hyphessobrycon heterorhabdus (Ulrey, 1894) \\ Hyphessobrycon sp. \\ Iguanodectes rachovii Regan, 1912 \\ Metynnis sp. \\ Moenkhausia chrysargyrea (Günther, 1864) \\ Moenkhausia collettii (Steindachner, 1882) \\ Poptella sp.
}


CORRÊA, J. M.; GERHARD, P.; FIGUEIREDO, R. O. Ictiofauna de igarapés de pequenas bacias de drenagem em área agrícola do Nordeste Paraense, Amazônia Oriental. Ambi-Agua, Taubaté, v. 7, n. 2, p. 214-230, 2012. (http://dx.doi.org/10.4136/ambi-agua.739)

Família CURIMATIDAE

Curimatopsis crypticus Vari, 1982

Curimatopsis sp.

Família ERYTHRINIDAE

Hoplias malabaricus (Bloch, 1794)

Família LEBIASINIDAE

Copella cf. arnoldi (Regan, 1912)

Copella cf. eigenmanni (Regan, 1912)

Nannostomus beckfordi Günther, 1872

Nannostomus eques Steindachner, 1876

Nannostomus trifasciatus Steindachner, 1876

Nannostomus sp.

Pyrrhulina cf. laeta (Cope, 1872)

Pyrrhulina sp.

Ordem GYMNOTIFORMES

Família APTERONOTIDAE

Apteronotus sp.

Ordem SILURIFORMES

Família HEPTAPTERIDAE

Mastiglanis asopos Bockmann, 1994

Família CALLICHTHYIDAE

Corydoras sp.

Família LORICARIIDAE

Farlowella sp.

Ordem BELONIFORMES

Família BELONIDAE

Potamorrhaphis guianensis (Jardine, 1843)

Ordem CYPRINODONTIFORMES

Família RIVULIDAE

Rivulus sp.

Ordem SYNBRANCHIFORMES

Família SYNBRANCHIDAE

Synbranchus marmoratus Bloch, 1795

Ordem PERCIFORMES

Família CICHLIDAE

Acaronia nassa (Heckel, 1840)

Aequidens sp.

Apistogramma caetei Kullander, 1980

Crenicara punctulatum (Günther, 1863)

Crenicichla sp.

Satanoperca jurupari (Heckel, 1840)

A ordem Characiformes foi a responsável pela maior parte dos exemplares capturados, com 1.920 indivíduos. Essa ordem apresentou a maior quantidade de espécies coletadas, 30 no total, sendo distribuídas em cinco famílias. A segunda ordem com maior representatividade foi Perciformes, que apresentou 121 exemplares com seis espécies, sendo todos da família Cichlidae, perfazendo um total de 5,72\% do total capturado (Tabela 2). 
CORRÊA, J. M.; GERHARD, P.; FIGUEIREDO, R. O. Ictiofauna de igarapés de pequenas bacias de drenagem em área agrícola do Nordeste Paraense, Amazônia Oriental. Ambi-Agua, Taubaté, v. 7, n. 2, p. 214-230, 2012. (http://dx.doi.org/10.4136/ambi-agua.739)

Tabela 2. Ordem, número de indivíduos $(\mathrm{N})$ e contribuição de cada ordem $(\mathrm{N} \%)$.

\begin{tabular}{l|rc}
\hline \multicolumn{1}{c|}{ Ordem } & $\mathbf{N}$ & $\mathbf{N}(\boldsymbol{\%})$ \\
\hline Characiformes & 1920 & 90,69 \\
Perciformes & 121 & 5,72 \\
Siluriformes & 49 & 2,31 \\
Beloniformes & 12 & 0,57 \\
Cyprinodontiformes & 12 & 0,57 \\
Gymnotiformes & 2 & 0,09 \\
Synbranchiformes & 1 & 0,05 \\
\hline Total & $\mathbf{2 1 1 7}$ & $\mathbf{1 0 0 , 0 0}$ \\
\hline
\end{tabular}

As espécies de peixes que foram capturadas não seguem o padrão Neotropical de distribuição de espécies em ordens, com maior predomínio de peixes Characiformes, seguido de Siluriformes, Gymnotiformes e Perciformes (Lowe-McConnell, 1999). Verificou-se que a ordem Characiformes foi bem capturada, seguida pela ordem Perciformes. Porém as ordens Siluriformes e Gymnotiformes foram pouco representadas. É possível que elas tenham sido subamostradas devido, provavelmente, à intensidade com que cada estação foi amostrada, à quantidade de apetrechos de pesca e sua seletividade e horário das amostragens. Por exemplo, o fato das coletas terem sido realizadas somente no período diurno contribui para que os Siluriformes fossem pouco representados.

Verificou-se que a ordem Characiformes foi predominante, sendo 30 espécies, num total de 1920 indivíduos $(90,69 \%$ da amostra) pertencentes a cinco famílias: Anostomidae, Characidae, Curimatidae, Erythrinidae e Lebiasinidae. Barbosa et al. (2003) realizando um estudo em um igarapé de terra firme situado na Amazônia Central, verificaram a presença de 10 espécies, distribuídos em 10 gêneros, sete famílias e quatro ordens. Os mesmos constataram que Characiformes foi a ordem predominante, com quatro espécies pertencentes a duas famílias.

\subsection{Constância de espécies}

Levando em consideração todas as 72 amostras realizadas, a espécie Iguanodectes rachovii ocorreu em 50\% das amostras, seguido da espécie Hyphessobrycon sp. com 45,8\% das amostras. A terceira maior ocorrência foi da espécie Pyrrhulina cf. laeta, em 40,3\% das amostras, seguido de Hyphessobrycon heterorhabdus e Bryconops melanurus, com 33,3\% das ocorrências. Oito espécies ocorreram em apenas uma das coletas efetuadas, contribuindo com $1,4 \%$ cada (Figura 2 ).

No presente estudo, das oito estações de amostragem, seis são ambientes característicos de poços, onde foi observada menor constância das espécies. É importante ressaltar que, embora não haja bibliografia de peixes amazônicos para comparar, a distribuição e constância de captura em diferentes habitats pode estar relacionadas com características hidrodinâmicas (vazão e corrente), aparato e horário de captura, características ambientais (vegetação aquática, disponibilidade de alimento, etc. 


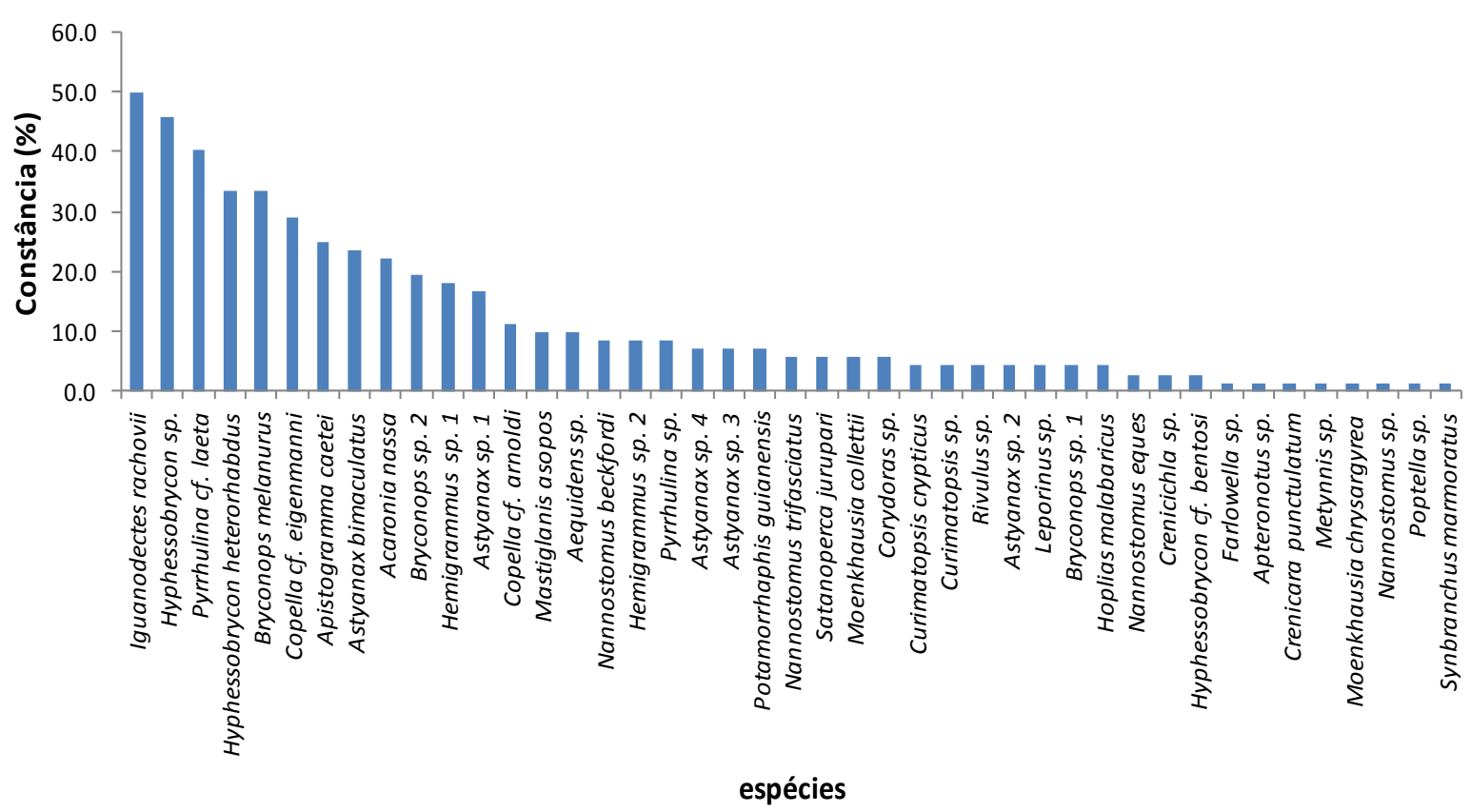

Figura 2. Constância das espécies em todas as oito estações de amostragem nos igarapés Cumaru, São João e Pachibá.

\subsection{Riqueza de espécies}

O Igarapé Pachibá, na estação de amostragem IGPA-B, apresentou a maior riqueza, com 21 espécies, sendo cinco espécies capturadas apenas nesse local: Curimatopsis crypticus, Curimatopsis sp., Nannostomus eques, Nannostomus sp. e Apteronotus sp. O Igarapé Cumaru, na estação IGCU-C apresentou 20, sendo Moenkhausia chrysargyrea apenas encontrada nesse ponto. O Igarapé Cumaru, na estação IGCU-D, apresentou 19 espécies e três capturadas neste lugar: Crenicara punctulatum, Farlowella sp. e Poptella sp. No Igarapé São João, na estação IGSJ-B, foram capturadas 18 espécies, sendo duas encontradas nesse ponto: Metynnis sp. e Rivulus sp. Os outros pontos de coleta que contribuíram com pouca riqueza de espécies foram: São João (IGSJ-A), com 13 espécies; Pachibá (IGPA-A), com 10 espécies, sendo Synbranchus marmoratus encontrado apenas nesse ponto; Cumaru (IGCU-A) com nove espécies. O Cumaru, no ponto IGCU-B, apresentou a menor riqueza, com apenas oito espécies, sendo Hoplias malabaricus encontrado somente nesse local de amostragem (Figura 3).

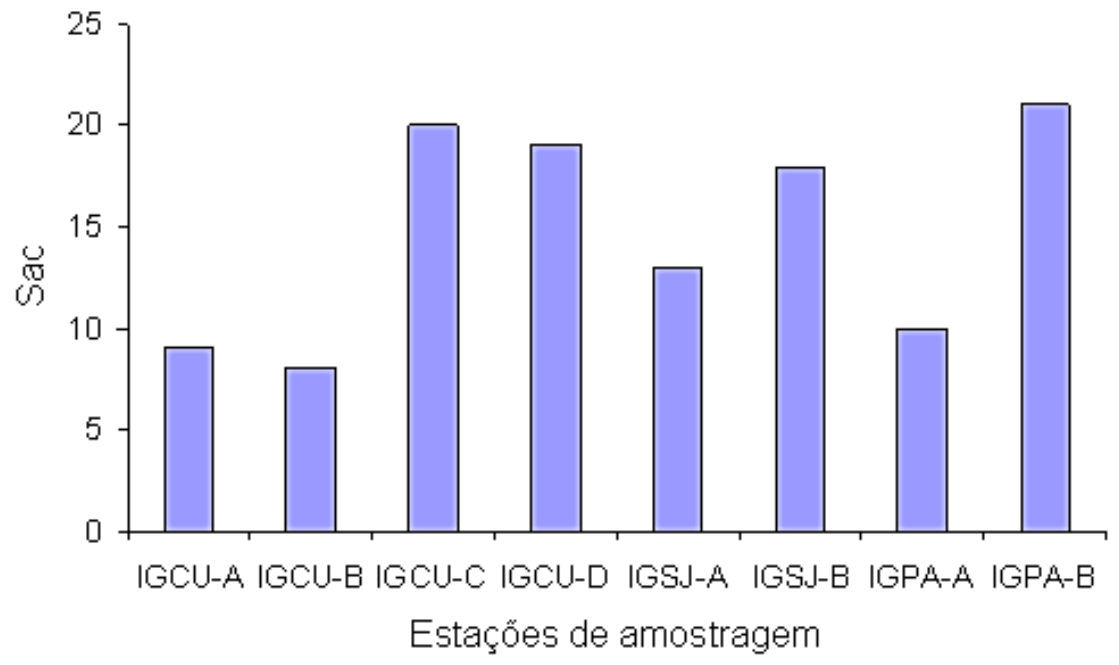

Figura 3. Número de espécies acumuladas $\left(S_{a c}\right)$ registradas em cada estação de amostragem. 


\subsection{Abundância das espécies}

Considerando todas as amostragens, a espécie Hyphessobrycon heterorhabdus foi a mais abundante, com 337 indivíduos, seguido da espécie Bryconops melanurus, com 326 espécimes, Pyrrhulina cf. laeta com 283 indivíduos e Iguanodectes rachovii, com 241 espécimes. Essas espécies corresponderam a $56 \%$ do total de indivíduos capturados. Seis espécies foram registradas com apenas um indivíduo: Crenicara punctulatum, Metynnis sp., Moenkhausia chrysargyrea, Nannostomus sp., Poptella sp. e Synbranchus marmoratus (Figura 4).

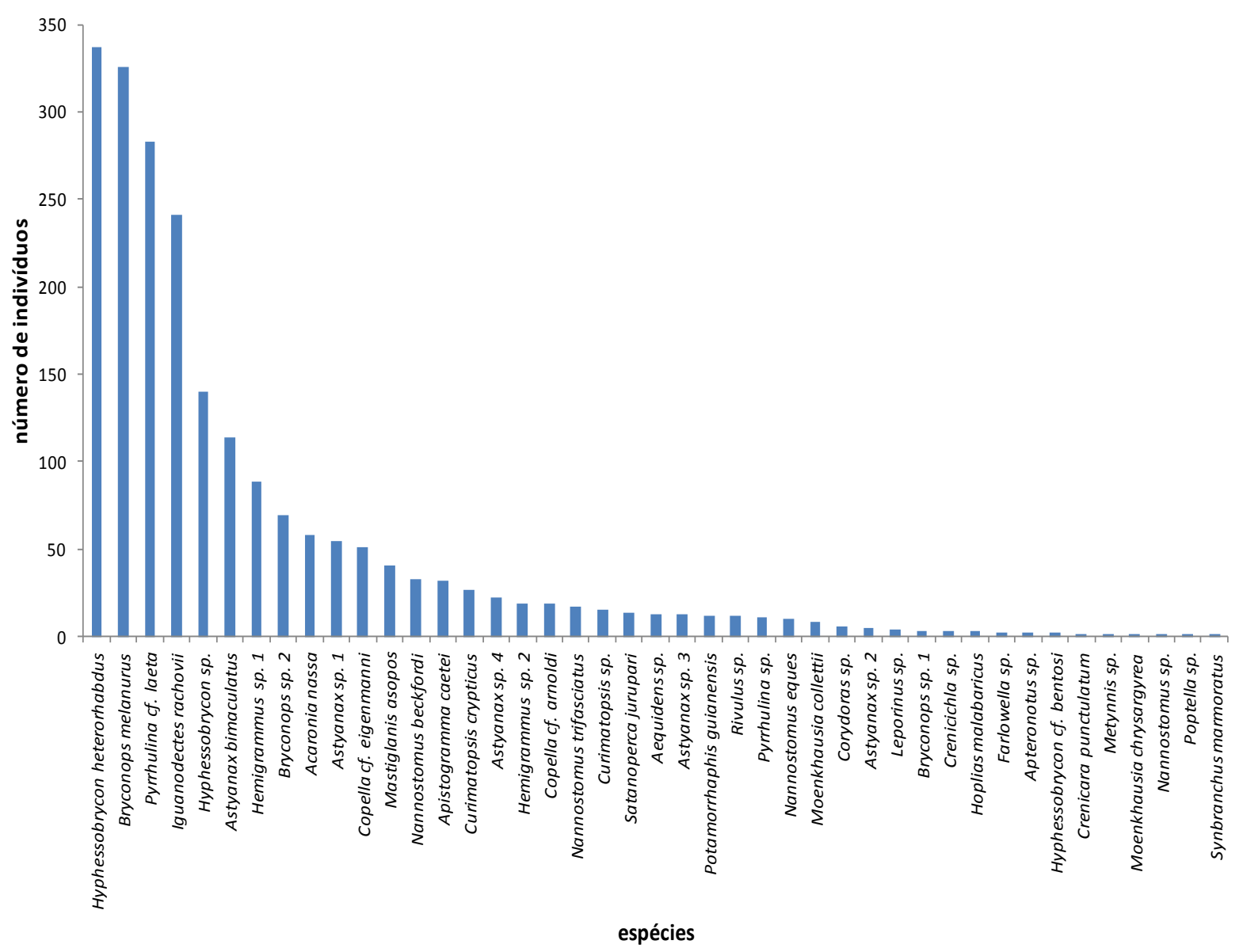

Figura 4. Ordenação de espécies por abundância de indivíduos nas oito estações de amostragem dos igarapés Cumaru, São João e Pachibá.

A abundância ou densidade relativa das espécies é um dos atributos ecológicos ou caracteres mais importantes nos estudos de comunidades de peixes, uma vez que mede a quantidade de peixes existentes, ou, na melhor das hipóteses, faz uma estimativa. Contudo, é também um dos parâmetros mais difíceis de se determinar na região Neotropical, principalmente em virtude da grande diversidade de formas e hábitos apresentados pelos peixes, o que faz com que as amostragens sejam viciadas, isto é, apresentem distorções causadas pelos métodos ou aparelhos usados nas capturas (Santos e Ferreira, 1999). Na maior parte de nossas amostragens, utilizou-se rede de arrasto e covos e, como estes apetrechos de pesca são bastante seletivos, geralmente selecionando as espécies de pequeno tamanho, outras espécies de peixes de tamanho médio não foram capturadas. A tarrafa também foi outro apetrecho de pesca seletivo, porém, neste caso foram capturadas indivíduos de médio porte. 
CORRÊA, J. M.; GERHARD, P.; FIGUEIREDO, R. O. Ictiofauna de igarapés de pequenas bacias de drenagem em área agrícola do Nordeste Paraense, Amazônia Oriental. Ambi-Agua, Taubaté, v. 7, n. 2, p. 214-230, 2012. (http://dx.doi.org/10.4136/ambi-agua.739)

\subsection{Medidas de diversidade}

A Tabela 3 apresenta as cinco diversas métricas (Número de espécies e indivíduos acumulados, Dominância, Diversidade e Equitabilidade) das comunidades de peixes estudadas. Dentre os pontos amostrados verificou-se que o IGCU-B apresentou uma comunidade de peixes com maior dominância $(D=0,43)$ e, consequientemente o menor valor de diversidade $\left(H^{\prime}=1,22\right)$, enquanto o IGPA-B apresentou o maior valor de diversidade $\left(H^{\prime}=2,39\right)$ e eqüitabilidade $(E q=0,78)$ e o menor valor de dominância $(D=0,12)$.

Tabela 3. Número de espécies acumuladas $\left(S_{a c}\right)$, número de indivíduos acumulados $\left(N_{a c}\right)$, Dominância de Simpson $(D)$, Diversidade de Shannon $\left(H^{\prime}\right)$ e Equitabilidade de Shannon $(E q)$ para os nove meses de coleta.

\begin{tabular}{l|ccccc}
\hline \multicolumn{1}{c|}{ Estações } & $\boldsymbol{S}_{\boldsymbol{a c}}$ & $\boldsymbol{N}_{\boldsymbol{a c}}$ & $\boldsymbol{D}$ & $\boldsymbol{H}^{\prime}$ & $\boldsymbol{E q}$ \\
\hline IGCU-A & 9 & 201 & 0,35 & 1,34 & 0,61 \\
IGCU-B & 8 & 397 & 0,43 & 1,22 & 0,59 \\
IGCU-C & 20 & 267 & 0,21 & 1,93 & 0,65 \\
IGCU-D & 19 & 294 & 0,39 & 1,62 & 0,55 \\
IGSJ-A & 13 & 328 & 0,20 & 1,92 & 0,75 \\
IGSJ-B & 18 & 163 & 0,16 & 2,18 & 0,75 \\
IGPA-A & 10 & 153 & 0,38 & 1,29 & 0,56 \\
IGPA-B & 21 & 314 & 0,12 & 2,39 & 0,78 \\
\hline Total & $\mathbf{4 3}$ & $\mathbf{2 1 1 7}$ & $\mathbf{0 , 0 9}$ & $\mathbf{2 , 7 7}$ & $\mathbf{0 , 7 4}$ \\
\hline
\end{tabular}

O Índice de Dominância de Simpson e a ordenação das espécies por abundância indicaram que o ponto B do igarapé Cumaru apresentou maior dominância, devido a maior abundância de Hyphessobrycon heterorhabdus sobre as demais espécies. Também nesse ponto de amostragem forma-se um ambiente de poço relativamente assoreado, com pouca cobertura e abrigo, sendo comum encontrar poucas espécies nesse local. Santos e Ferreira (1999) afirmam que a dominância relativamente alta de uma determinada espécie é devida à presença de cardumes ou de áreas muito modificadas pela atividade humana, como em igarapés e rios represados.

O Índice de Diversidade de Shannon revelou que o ponto IGPA-B do igarapé Pachibá possuiu maior diversidade do que os outros pontos de coleta. Isto se deve ao fato de que nessa estação de amostragem foi capturado o maior número de espécies acumuladas. Tal fato está associado à mudança no mesohabitat, pois, as três primeiras coletas nessa estação foram feitas a jusante do lago, sendo capturadas poucas espécies e que as coletas seguintes foram realizadas no próprio lago, sendo capturadas mais espécies.

Na microbacia do Igarapé Pachibá, a maior diversidade no trecho médio corrobora o Conceito de Rio como Contínuo ("River Continuum Concept"), de Vannote et al. (1980). Estes autores propuseram que a diversidade biológica em ambientes lóticos é máxima nos trechos médios destes sistemas. Barbosa et al. (2003), acharam maior índice de diversidade nas porções intermediárias do que nas nascentes. Os autores sugerem a existência de um gradiente longitudinal entre os ambientes, a qual pode estar relacionado com as barreiras físicas naturais, corredeiras e cachoeiras existentes ao longo do igarapé.

\subsection{Similaridade entre as comunidades}

A análise de agrupamento apresentou dois grupos no nível de 0,8 de dissimilaridade, denominados A e B (Figura 5). Este nível de corte corresponde exatamente aos grupos das 
amostras tomadas nas estações dos trechos médios (grupo A) e nascentes (grupo B). Estes grupos diferenciaram-se por um conjunto de espécies exclusivas, sendo Hemigrammus sp. $1 \mathrm{e}$ Bryconops sp. 2 as espécies exclusivas do grupo A e Copella cf. eigenmanni, Copella cf. arnoldi e Pyrrhulina sp. as espécies exclusivas do grupo B. Em relação à dominância por espécies, Bryconops melanurus foi a mais representativa no grupo A, enquanto Pyrrhulina cf. laeta obteve maior representatividade no grupo B.

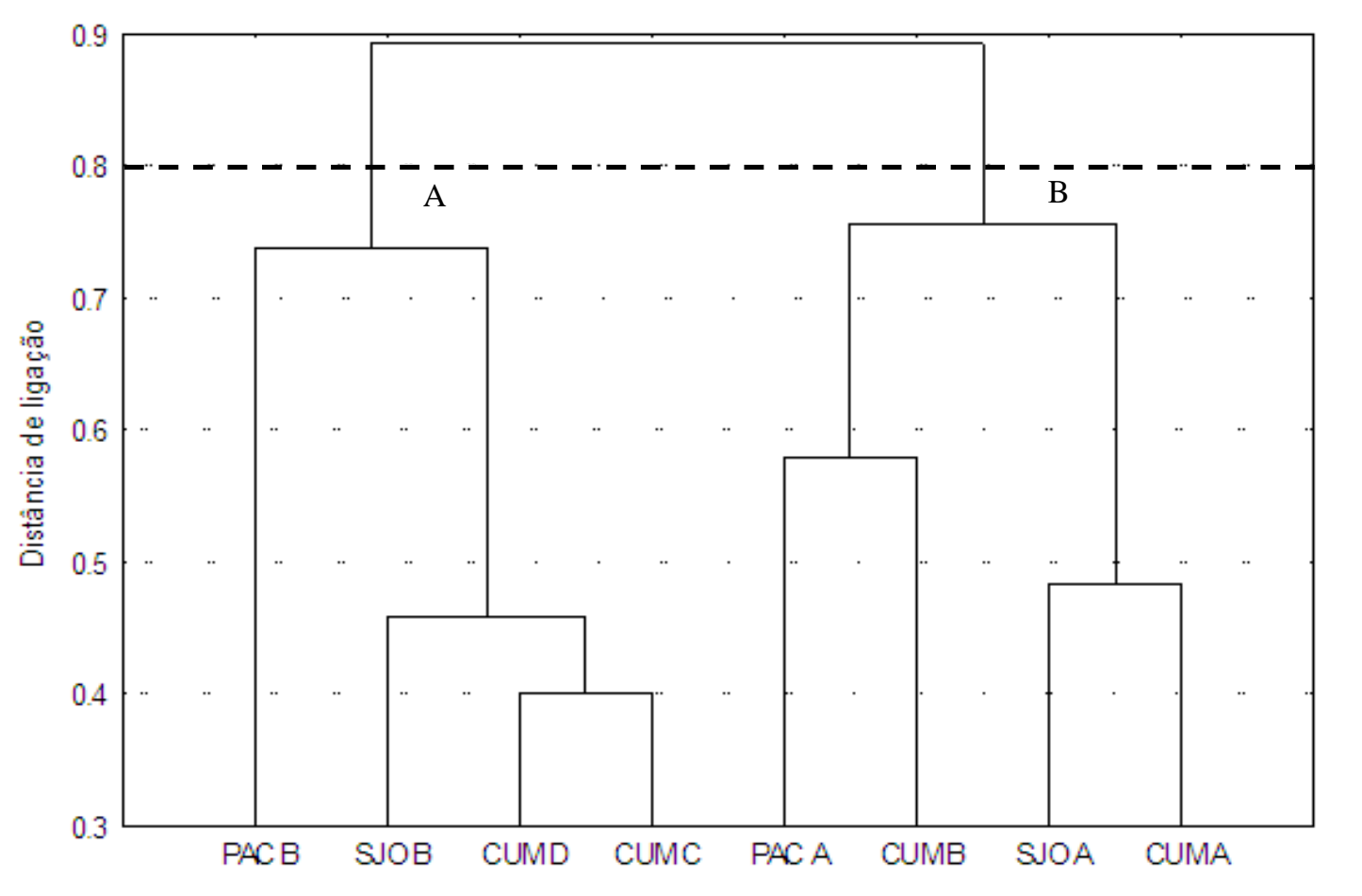

Figura 5. Resultado da análise de agrupamento entre as estações de amostragem. Corte ao nível de 0,8 de dissimilaridade.

Assim verificou-se que a distribuição das espécies nos igarapés seguiu um padrão longitudinal ao invés de um padrão geográfico (referente à bacia hidrográfica a que pertencem as microbacias), ou seja, independente do igarapé, a maioria das espécies que ocorreram nas nascentes não ocorreram nas porções intermediárias desses igarapés e vice-versa (Figura 5). Suarez e Petrere Júnior (2003) estudando associações de espécies de peixes em ambientes lóticos da bacia do rio Iguatemi, sugeriram que existe uma diferenciação no uso dos habitats pelas espécies de peixes que leva a uma formação de diferentes grupos de espécies coocorrentes e que esses grupos são representados por espécies característicos de riachos e por espécies característicos de trechos de rios.

No grupo A, a espécie Bryconops melanurus, além de ter sido uma espécie dominante, também foi uma das espécies exclusivas desse local. Isto se deve ao fato de que esse animal tem uma preferência por águas com leve correnteza e com substrato rochoso (Froese e Pauly, 2007). O gênero Copella, em especial a espécie Copella cf. eigenmanni destacou-se no grupo $\mathrm{B}$. Isto se deve provavelmente a estes indivíduos serem encontrados próximos às margens das nascentes, onde há uma redução considerável da correnteza e acúmulo de folhiços no substrato. As estações amostradas tinham predomínio total de ambientes represados, tendendo ao lêntico. 
CORRÊA, J. M.; GERHARD, P.; FIGUEIREDO, R. O. Ictiofauna de igarapés de pequenas bacias de drenagem em área agrícola do Nordeste Paraense, Amazônia Oriental. Ambi-Agua, Taubaté, v. 7, n. 2, p. 214-230, 2012. (http://dx.doi.org/10.4136/ambi-agua.739)

\subsection{Análise de regressão linear}

Não houve diferença significativa entre o número de espécies acumuladas com o uso da terra (Tabela 4).

Tabela 4. Valores da regressão linear entre o número de espécies acumuladas $\left(\mathrm{S}_{\mathrm{ac}}\right)$ e as variáveis do uso da terra.

\begin{tabular}{l|cc}
\hline & $\mathbf{r}^{2}$ & $\mathbf{p}$ \\
\hline $\mathrm{S}_{\mathrm{ac}}$ X Agricultura & 0,289 & 0,1677 \\
$\mathrm{~S}_{\mathrm{ac}}$ X Pasto & 0,328 & 0,1359 \\
$\mathrm{~S}_{\mathrm{ac}}$ X Capoeira & 0,217 & 0,2444 \\
$\mathrm{~S}_{\mathrm{ac}}$ X Floresta & 0,313 & 0,1480 \\
$\mathrm{~S}_{\mathrm{ac}}$ X Agricultura (\%) & 0,369 & 0,1083 \\
$\mathrm{~S}_{\mathrm{ac}}$ X Pasto (\%) & 0,149 & 0,3465 \\
$\mathrm{~S}_{\mathrm{ac}}$ X Capoeira $(\%)$ & 0,268 & 0,1866 \\
$\mathrm{~S}_{\mathrm{ac}}$ X Floresta $(\%)$ & 0,351 & 0,1195 \\
\hline
\end{tabular}

Não foi observada uma diferença significativa entre o número de indivíduos acumulados e índice de dominância de Simpson com as variáveis do uso da terra (Tabelas 5 e 6).

Tabela 5. Valores da regressão linear entre o número de indivíduos acumulados $\left(\mathrm{N}_{\mathrm{ac}}\right)$ e as variáveis do uso da terra.

\begin{tabular}{l|cc}
\hline & $\mathbf{r}^{2}$ & $\mathbf{p}$ \\
\hline $\mathrm{N}_{\mathrm{ac}}$ X Agricultura & 0,002 & 0,9107 \\
$\mathrm{~N}_{\mathrm{ac}}$ X Pasto & 0,018 & 0,7466 \\
$\mathrm{~N}_{\mathrm{ac}}$ X Capoeira & 0,000 & 0,9478 \\
$\mathrm{~N}_{\mathrm{ac}}$ X Floresta & 0,001 & 0,9331 \\
$\mathrm{~N}_{\mathrm{ac}}$ X Agricultura (\%) & 0,007 & 0,8378 \\
$\mathrm{~N}_{\mathrm{ac}}$ X Pasto $(\%)$ & 0,087 & 0,5184 \\
$\mathrm{~N}_{\mathrm{ac}}$ X Capoeira (\%) & 0,071 & 0,5260 \\
$\mathrm{~N}_{\mathrm{ac}}$ X Floresta (\%) & 0,088 & 0,5212 \\
\hline
\end{tabular}

Tabela 6. Valores da regressão linear entre o Índice de Dominância de Simpson (D) e as variáveis do uso da terra.

\begin{tabular}{l|cc}
\hline & $\mathbf{r}^{2}$ & $\mathbf{p}$ \\
\hline D X Agricultura & 0,029 & 0,6880 \\
D X Pasto & 0,011 & 0,7996 \\
D X Capoeira & 0,235 & 0,2221 \\
D X Floresta & 0,016 & 0,7610 \\
D X Agricultura (\%) & 0,099 & 0,5483 \\
D X Pasto (\%) & 0,077 & 0,5106 \\
D X Capoeira (\%) & 0,093 & 0,5327 \\
D X Floresta (\%) & 0,031 & 0,6743 \\
\hline
\end{tabular}


O número acumulado de espécies, o número acumulado de indivíduos e o Índice de Dominância de Simpson não foram influenciados pelas variáveis relacionadas ao uso da terra, ou seja, valores absolutos e percentuais de agricultura, pasto, capoeira e floresta. Assim sendo, apesar de algumas das estações de amostragem apresentarem processo inicial de assoreamento, o uso da terra predominante, a agricultura familiar, na abrangência espacial e intensidade que vem sendo praticada nas microbacias estudadas, ainda propicia que as maiores áreas das pequenas propriedades rurais mantenham-se cobertas por floresta secundária de diferentes idades, principalmente nas zonas ripárias, possibilitando assim que o ecossistema aquático seja habitado por diversas espécies de peixes característicos de igarapés.

No entanto, em outras regiões, como por exemplo, no sudeste brasileiro, a utilização da terra provocou um desequilíbrio nas comunidades de peixes. Casatti (2004) estudando a ictiofauna de dois riachos no alto rio Paraná, sendo um deles assoreado em área de pastagem e sem a vegetação ripária e o outro de referência, com a vegetação ripária, verificou a presença de 21 espécies de peixes no córrego assoreado. Destacou que a espécie Pyrrhulina cf. laeta foi uma das espécies dominantes favorecidas por habitats relacionados ao assoreamento, com ocorrência de 50\% nesse ambiente.

\subsection{Curva de acumulação}

Considerando todas as amostragens, verificou-se que a curva de acumulação não apresentou tendência à estabilização (Figura 6). Isto indica que o número total de espécies que ocorrem nesses ambientes ainda não foi alcançado. É importante que esse tipo de estudo tenha continuidade.

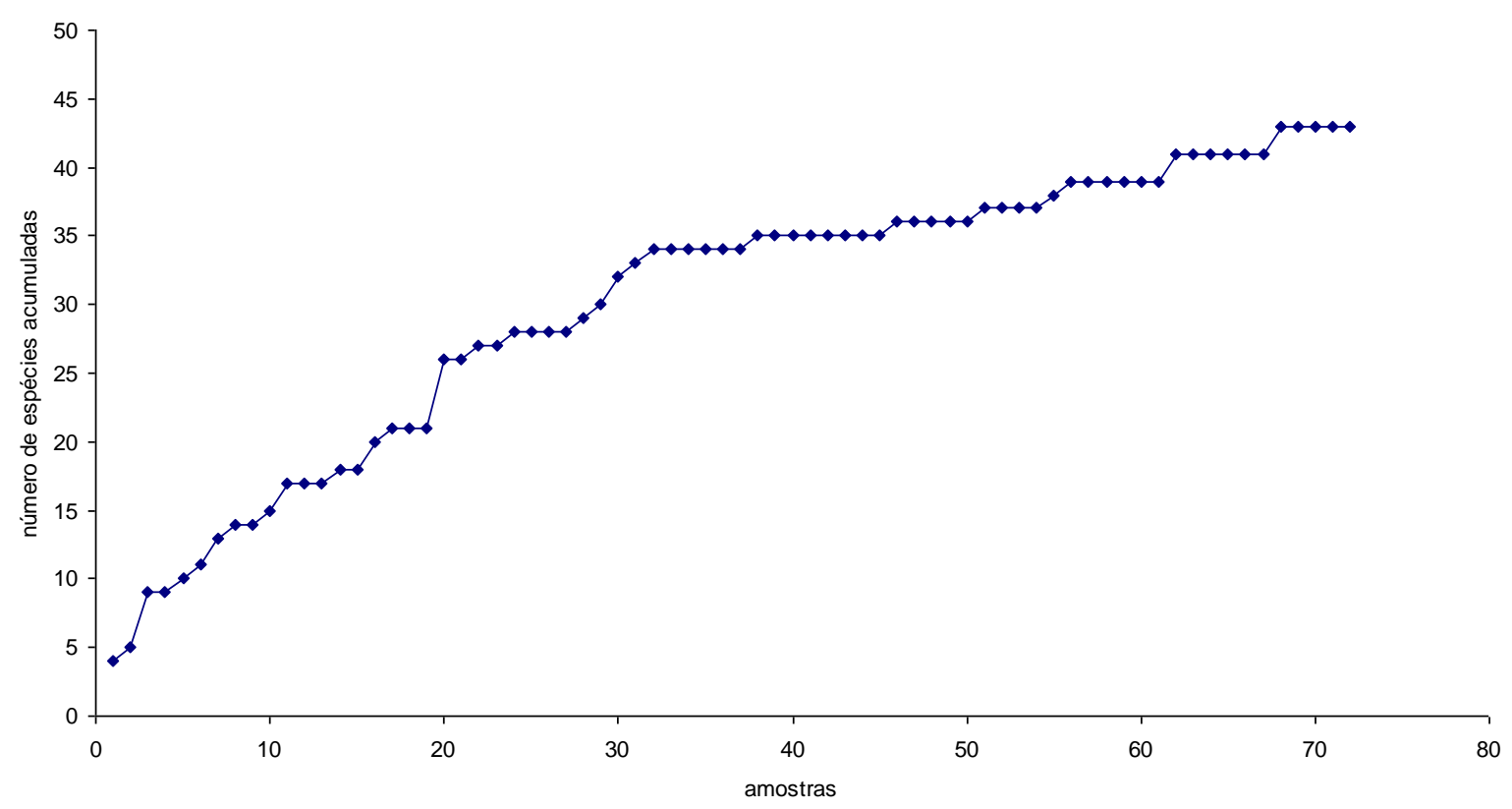

Figura 6. Curva de acumulação de espécies para todas as estações de amostragem nos igarapés Cumaru, São João e Pachibá.

\section{CONCLUSÃO}

Os trechos médios, com base nos índices de diversidade calculados, apresentaram uma ictiofauna com maior diversidade de espécies, mostrando que a diversidade aumenta de acordo com o tamanho do habitat ao longo dos igarapés.

A distribuição dos peixes seguiu um padrão longitudinal ao invés de um padrão geográfico, com predominância de espécies exclusivas tanto nas nascentes quanto nas porções 
CORRÊA, J. M.; GERHARD, P.; FIGUEIREDO, R. O. Ictiofauna de igarapés de pequenas bacias de drenagem em área agrícola do Nordeste Paraense, Amazônia Oriental. Ambi-Agua, Taubaté, v. 7, n. 2, p. 214-230, 2012. (http://dx.doi.org/10.4136/ambi-agua.739)

intermediárias. A agricultura familiar, da maneira como se processa atualmente na região, pareceu não influenciar grandemente na estrutura das comunidades de peixes, já que a intensidade com que essa atividade vem sendo executada ainda possibilita que o ecossistema aquático seja habitado por diversas espécies de peixes.

\section{AGRADECIMENTOS}

Aos projetos Agrobacias Amazônicas (EMBRAPA, 02.02.5.32), Rede Tipitamba (EMBRAPA, 02.03.1.10), Aguamor (CT-Hidro/CNPq, 502626/2003-8), e Milênio LBA2 (Institutos do Milênio/CNPq, 420199/2005-5), e respectivas equipes, pelo apoio na execução desse trabalho. A Universidade Federal Rural da Amazônia, por ter concedido espaço físico para as análises de identificação. Aos funcionários e estagiários da Embrapa Amazônia Oriental, em especial ao técnico Sr. Reginaldo Ramos Frazão, pelo apoio no trabalho de campo.

\section{REFERÊNCIAS}

ALLAN, J. D. Landscapes and riverscapes: the influence of land use on stream ecosystems. Annual Review of Ecology, Evolution and Systematics, v. 35, p .257-284, 2004. http://dx.doi.org/10.1146/annurev.ecolsys.35.120202.110122

BARBOSA, R. P.; FREITAS, C. E. C.; SANTOS, S. M. The fish community of an upland stream in the Central Amazon (Presidente Figueiredo - Amazonas - Brazil). Acta Limnologica Brasiliensia, Rio Claro, v. 15, n. 2, p. 37-41, 2003.

BASTOS, T.; PACHECO, N. A. Rainfall distribution and water balance for day periods in Igarapé-Açu, Eastern Amazonia, Brazil. In: GERMAN-BRAZILIAN WORKSHOP ON NEOTROPICAL ECOSYSTEMS - ACHIEVEMENTS AND PROSPECTS OF COOPERATIVE RESEARCH, 2000, Hamburg. Abstracts... Hamburg: Universität Hamburg, 2000. p. 213.

BUCKUP, P. A.; MENEZES, N. A. (Eds.). Catálogo dos peixes marinhos e de água doce do Brasil. 2. ed. 2003. Disponível em: <http://www.mnrj.ufrj.br/catalogo>. Acesso em: 01 jul. 2007.

BUSS, D. F.; BAPTISTA, D. F.; SILVEIRA, M. P.; NESSIMIAN, J. L.; DORVILLÉ, L. F. $M$. Influence of water chemistry and environmental degradation on macroinvertebrate assemblages in a river basin in south-east Brazil. Hydrobiologia, v. 481, n. 1-3, p. 125136, 2002. http://dx.doi.org/10.1023/A:1021281508709

CASATTI, L. Ichthyofauna of two streams (silted and reference) in the upper Paraná river basin, southeastern Brazil. Brazilian Journal of Biology, São Carlos, v. 64, n. 4, p. 757-765, 2004. http://dx.doi.org/10.1590/S1519-69842004000500004

CASATTI, L. Alterações no Código Florestal Brasileiro: impactos potenciais sobre a ictiofauna. Biota Neotropica, Campinas, v. 10, n. 4, p. 31-34, 2010. Disponível em: <http://www.biotaneotropica.org.br/v10n4/en/abstract?article+bn00310042010>. Acesso em: 03 abr. 2012.

CHERNOFF, B. A.; MACHADO-ALLISON, A.; SAUL, W. La conservación de los ambientes acuáticos: una necesidad impostergable. Acta Biologica Venezuelica, Caracas, v. 16, n. 2, p. 1-3, 1996. 
DE PAULA, F. R.; FERRAZ, S. F. B.; GERHARD, P.; VETTORAZZI, C. A.; FERREIRA, A. Large woody debris input and its influence on channel structure in agricultural lands of southeast Brazil. Environmental Management, v. 48, n. 4, p. 750-763, 2011. http://dx.doi.org/10.1007/s00267-011-9730-4

FERREIRA, C. P.; CASATTI, L. Integridade biótica de um córrego na bacia do Alto Rio Paraná avaliada por meio da comunidade de peixes. Biota Neotropica, Campinas, v. 6, n. 3, 2006. Disponível em: <http://www.biotaneotropica.org.br/v6n3/pt/abstract? article+bn00306032006ISSN1676-0603>. Acesso em: 03 abr. 2012.

FERREIRA, E. J. G.; ZUANON, J. A. S.; SANTOS, G. M. Peixes comerciais do médio amazonas: região de Santarém, Pará. Brasília: IBAMA, 1998. 214 p.

FROESE, R.; PAULY, D. (Eds.). Fishbase. Version 08/2007. World Wide Web electronic publication. Disponível em: 〈http://www.fishbase.org/search.php>. Acesso em: 15 set. 2007.

GERHARD, P. Definição de Índices de Integridade Biótica no meio aquático em bacias hidrográficas da microrregião Bragantina (PA), como subsídio para aferição da sustentabilidade de sistemas de produção na agricultura familiar. Belém: Embrapa Amazônia Oriental, 2007. 53 p.

KREBS, C. J. Ecological methodology. New York: Benjamin/Cummings, 1999. 620 p.

LOWE-McCONNELL, R. H. Estudos ecológicos de comunidades de peixes tropicais. São Paulo: EDUSP, 1999. 536 p.

MACHADO, N. G.; VENTICINQUE, E. M.; PENHA, J. Effect of environmental quality and mesohabitat structure on a Biotic Integrity Index based on fish assemblages of cerrado streams from Rio Cuiabá basin, Brazil. Brazilian Journal of Biology, São Carlos, v. 71, n. 3, p. 577-586, 2011. http://dx.doi.org/10.1590/S1519-69842011000400002

PACHÊCO, N. A.; BASTOS, T. X. Boletim agrometeorológico 2006 Igarapé-Açu. Belém: Embrapa Amazônia Oriental, 2007. 32 p.

REIS, R. E.; KULLANDER, S. O.; FERRARIS, C. J. The check list of the freshwater fishes of South and Central America. Porto Alegre: EDIPUCRS, 2003. 944 p.

SANTOS, G. M.; FERREIRA, E. J. G. Peixes da Bacia Amazônica. In: LOWEMcCONNELL, R. H. Estudos ecológicos de comunidades de peixes tropicais. São Paulo: EDUSP, 1999. p. 345-373.

SANTOS, G. M.; MERONA, B.; JURAS, A. A.; JEGU, M. Peixes do baixo rio Tocantins: 20 anos depois da usina hidrelétrica Tucuruí. Brasília: ELETRONORTE, 2004. 216 p.

SILVANO, R.; OYAKAWA, O.; AMARAL, B.; BEGOSSI, A. Peixes do alto rio Juruá (Amazonas, Brasil). São Paulo: EDUSP, 2001. 302 p.

SOMMER, R.; VLEK, P. L. G.; SÁ, T. D. A.; VIELHAUER, K.; COELHO, R. F. R.; FÖLSTER, H. 2004. Nutrient balance of shifting cultivation by burning or mulching in the Eastern Amazon - evidence for subsoil nutrient accumulation. Nutrient Cycling in Agroecosystems, v. 68, n. 3, p. 257-271, 2004. http://dx.doi.org/10.1023/B:FRES.0000019470.93637.54 
SUAREZ, Y. R.; PETRERE JÚNIOR, M. Associações de espécies de peixes em ambientes lóticos da bacia do rio Iguatemi, Estado do Mato Grosso do Sul. Acta Scientiarum, Maringá, v. 25, n. 2, p. 361-367, 2003. http://dx.doi.org/10.4025/actascibiolsci.v25i2.2024

VANNOTE, R. L.; MINSHALL, K. W.; CUMMINS, K. W.; SEDELL, J. R.; CUSHING, C. E. The river continuum concept. Canadian Journal of Fisheries and Aquatic Sciences, v. 37, p. 130-137, 1980. http://dx.doi.org/10.1139/f80-017

WATRIN, O. S.; GERHARD, P.; MACIEL, M. N. M. Dinâmica do uso da terra e configuração da paisagem em antigas áreas de colonização de base econômica familiar, no Nordeste do Estado do Pará. Geografia, Rio Claro, v. 34, p. 455-472, 2009. 